\title{
Asthma and hyperbilirubinemia: a new aspect to analyze?
}

\author{
Eliza Wasilewska ${ }^{1}$, Barbara Kaczorowska-Haćc,3, Beata Burzyńska ${ }^{4}$, Sylwia Małgorzewicz $^{5}$, Ewa Jassem ${ }^{1}$
}

${ }^{1}$ Department of Allergology, Medical University of Gdańsk, Gdańsk, Poland

${ }^{2}$ Department of Occupational Therapy, Gdańsk University of Physical Education and Sport, Gdańsk, Poland

${ }^{3}$ Paediatric, Haematology Department, Medical University of Gdańsk, Gdańsk, Poland

${ }^{4}$ Institute of Biochemistry and Biophysics, Polish Academy of Sciences, Warsaw, Poland

${ }^{5}$ Department of Clinical Nutrition, Medical University of Gdańsk, Gdańsk, Poland

Adv Dermatol Allergol 2019; XXXVI (5): 639-642 DOI: https://doi.org/10.5114/ada.2019.89511

One of the concepts of airway inflammation in asth$\mathrm{ma}$ is the occurrence of oxidative stress defined as the disruption of the balance between the level of oxidants and reductants (antioxidants). Several factors, such as cigarettes, marijuana smoking, air pollution, stress, improper dietary habits e.g. eating processed food with a high content of preservatives, can increase the severity of oxidative stress. Conversely, bilirubin is considered to be a factor having cytoprotective properties which include antioxidant, anti-inflammatory, and antiproliferative effects [1]. However, its total effect on the pulmonary system remains unknown.

We report the case of a 16-year-old boy, admitted to the Allergology Department due to ongoing $5 \mathrm{~h}$ of dyspnea and jaundice. The patient had a history of recurrent bronchitis from 3 to 5 years of age approximately 4 times a year, and less from the age of $6-2$ times a year, but bronchitis was accompanied by prolonged cough of up to 4 weeks. He has poor tolerance of exercise from the age of 10, and reported symptoms which included coughing, wheezing, dyspnea and fatigue especially after physical exercises and swimming. In addition, he sometimes experiences loss of appetite and had also accidentally noticed jaundice on the same days, when he had coughing and wheezing. The patient's parents attributed the symptoms to intensive studying and examination-related stress, and poor dietary habits as he often ate processed food such as chips and cola. The patient had a history of hyperbilirubinemia (14 mg/dl) at birth, however no further diagnosis had been pursued at the time. Since then, the level of bilirubin has never been marked. The patient had a family history of chronic diseases, negated having alcohol intake, or drug abuse. On physical examination, superficial jaundice, dyspnea, wheezing and pharyngeal erythema were noted. His full blood count values, $C$ protein $(1.00 \mathrm{mg} / \mathrm{l})$, reticulocytes
(0.5\%), iron concentration $(87 \mu \mathrm{g} / \mathrm{dl})$, transferrin saturation (32\%), liver function tests (ALT, AST, gGT) were normal despite unconjugated bilirubin $(7.49 \mathrm{mg} / \mathrm{dl})$. Tests for lambliasis (stool microscopy and antigen testing), AgHBs and HCV antibodies were negative, abdominal ultrasonography was normal. The pulmonary function test (Spirometer Jaeger) revealed: forced expiratory volume in $1 \mathrm{~s}\left(\mathrm{FEV}_{1}\right) 63 \%$, predicted value (PV), forced vital capacity (FVC) $78 \%$, PV, FEV $/$ FVC - 68\%. The challenge test with $400 \mu \mathrm{g}$ of salbutamol was positive $\left(\Delta \mathrm{FEV}_{1}-\right.$ $28.4 \%, \Delta \mathrm{FVC} 210 \mathrm{ml}$ ). He was intensively treated with nebulization with short-acting $\beta$, mimetics and steroids (budesonide), with a good effect, shortness of breath, subsided after a few hours. Afterwards, treatment with nebulization was continued and it was noted that dyspnea, wheezing disappeared, presenting jaundice had reduced. Follow-up laboratory tests performed 5 days later showed normal bilirubin concentration (1.1 mg/dl), total IgE level $46.35 \mathrm{kU} / \mathrm{l}$, specific lgE to inhalant allergens undetectable despite cockroach allergen (53.70 kU/l). Skin prick tests (Allergopharma, Germany) were positive only for cockroach allergen. After 3 days of nebulization, the boy was treated with short-acting $\beta$, mimetics and steroids (ciclesonide $160 \mathrm{mg}$ twice daily) in an inhaled form and oral leukotriene modifiers $10 \mathrm{mg}$ daily. After 3 months his exercise tolerance improved, spirometry values improved, but the challenge test was positive.

Due to the high levels of bilirubin and jaundice, genetic testing for Gilbert's syndrome (GS) was carried out. Genomic DNA was isolated from peripheral blood samples and the patient was found to be homozygous for the UDPglucuronosyltransferase 1A1 (UGT1A1) promoter mutation characteristic of GS (Figures 1, 2). Owing to the fact that the bilirubin levels were relatively higher than those associated with GS, we also decided to test for HFE mutations and H63D mutation carriage was confirmed (Figures 3, 4).

Address for correspondence: Eliza Wasilewska MD, PhD, Department of Allergology, Medical University of Gdansk, 7 Debinki St, 80-211 Gdańsk, Poland, phone: +48 5834927 24, fax: +48 5834927 23, e-mail: ewasilewska@gumed.edu.pl Received: 24.01.2018, accepted: 4.06.2018. 

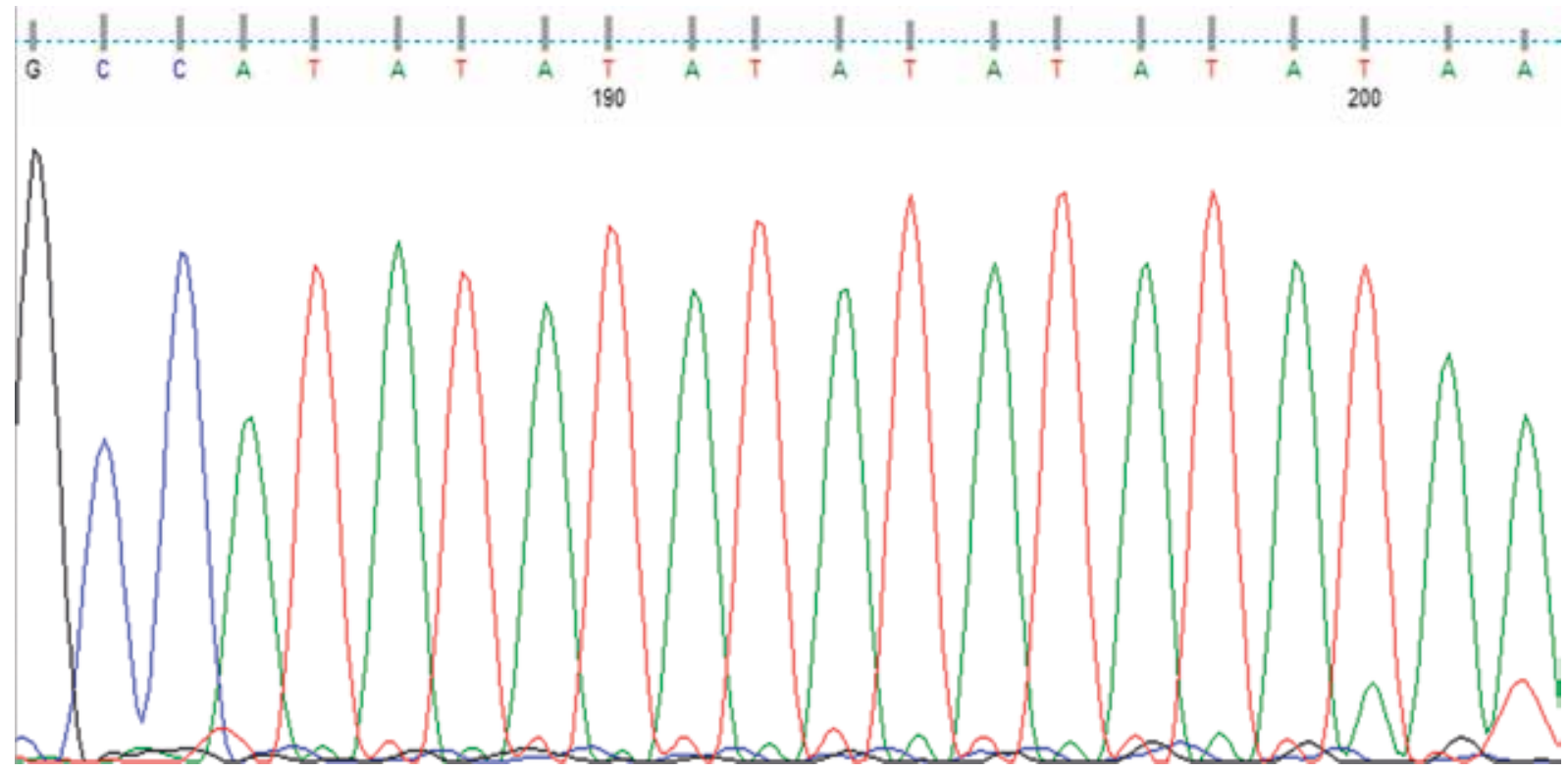

Figure 1. Results of Gilbert's syndrome mutation analysis - patient (TA)7/(TA)7. Genomic DNA was isolated from peripheral blood samples using the MagNA Pure Compact Nucleic Acid Isolation Kit I (Roche, Germany). The promoter region of the UGT1A1 gene was amplified by polymerase chain reaction (PCR) with F primer $5^{\prime}$ AAAGTGAACTCCCTGCTACC $3^{\prime}$ and R primer 5' TGCTCCTGCCAGAGGTT 3'. Cycling conditions comprised 30 cycles of denaturation at $95^{\circ}$ for $30 \mathrm{~s}$, annealing at $52^{\circ}$ for $30 \mathrm{~s}$, and extension at $72^{\circ}$ for $20 \mathrm{~s}$. The PCR fragments were sequenced directly in both directions with BigDye Terminators and appropriate primers using an ABI Prism 377 sequencer (Applied Biosystems, CA, USA). Sequencing of the promoter region of the UGT1A1 gene revealed a (TA)7/(TA)7 genotype and the patient was found to be homozygous for the UGT1A1 promoter mutation characteristic of Gilbert's syndrome

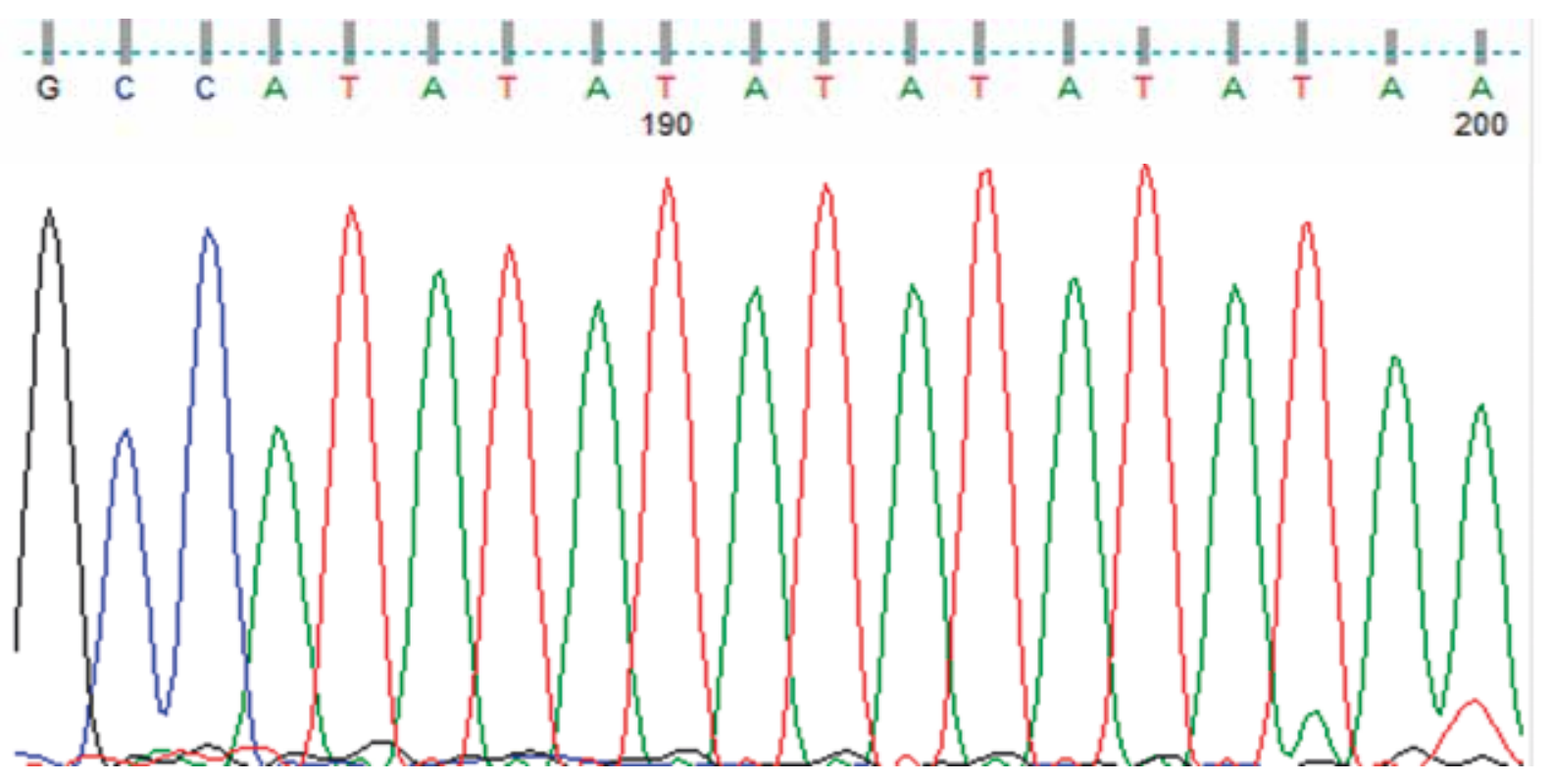

Figure 2. Results of Gilbert's syndrome mutation analysis - healthy control (TA)6/(TA)6 


\section{A}



B

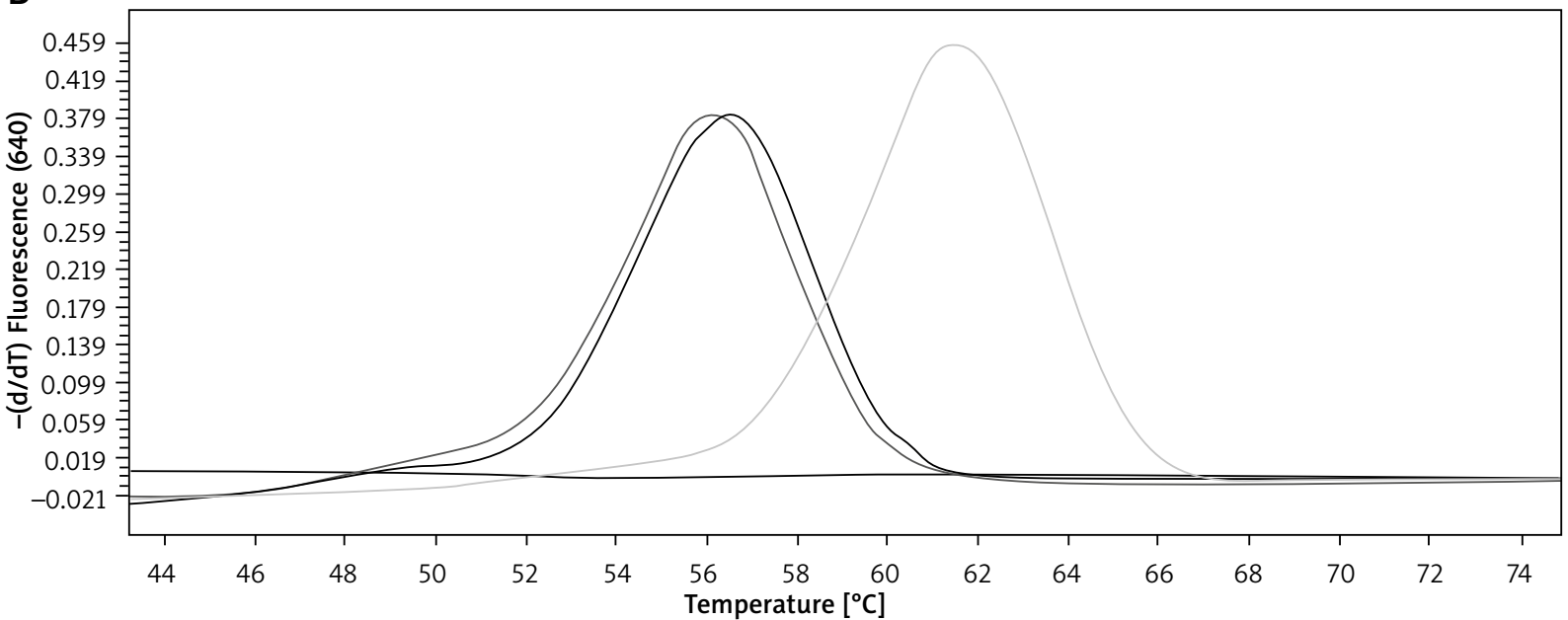

Figure 3. Genotyping of HFE gene mutation - patient with a HFE mutation (A - melting curves, B - melting peaks)

A

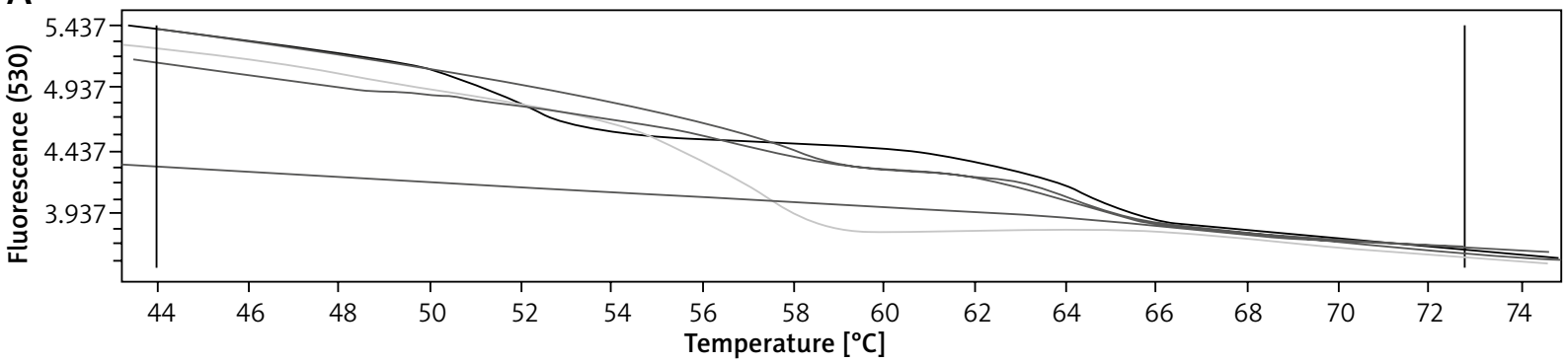

B

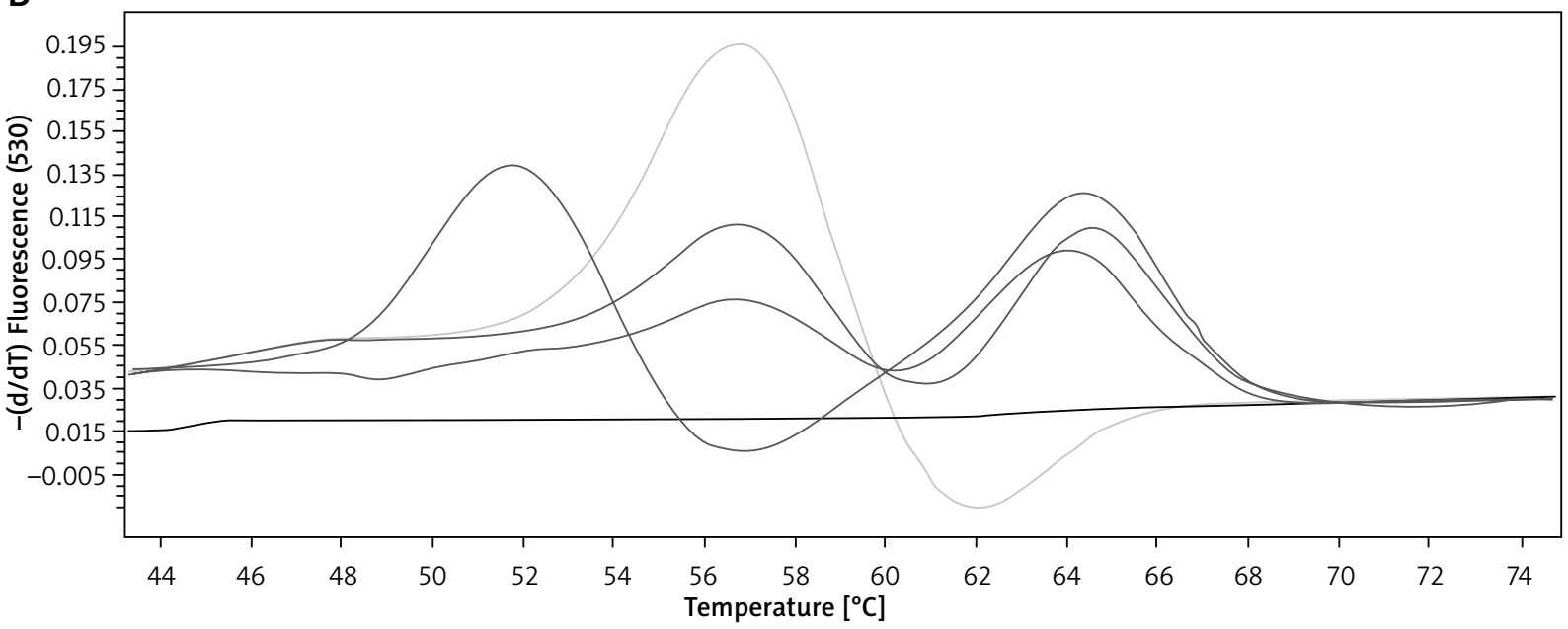

Figure 4. Genotyping of HFE gene mutation - healthy control 
Our final diagnosis was uncontrolled asthma, hyperreactivity to cockroach allergen, Gilbert's syndrome, and carriage of HFE mutation.

To our knowledge this is the first report of a patient with hyperbilirubinemia and asthma exacerbation. Experimental studies using animal models support a protective effect of bilirubin against respiratory injury by environmental factors [2, 3]. A similar positive role of bilirubin on pulmonary function and lower rates of respiratory diseases has been noted in some cohort studies $[4,5]$. Horsfall et al. concluded that patients predisposed to Gilbert's syndrome have higher pulmonary function (mean FEV and FVC) but the authors took into account only the genetic mutation and not the level of bilirubin in the blood [6]. GS is a genetic disease characterized by mild hyperbilirubinemia, with absence of liver disease or overt hemolysis, UGT1A1 activity $<35 \%$ of the normal value, caused by mutations in the UGT1A1 gene which encodes hepatic UGT1A1. GS occurs in $9 \%$ of the general population, and as a mild disorder does not require treatment. In another study, the same authors described a correlation between relatively high bilirubin levels within the normal range and a lower risk of COPD and lung cancer [5].

Despite the protective role of bilirubin on pulmonary function, our patient with hyperbilirubinemia presented with asthma. We concluded that coexistence of some other factors, such as stress, improper diet with low antioxidant agents, and exposure to chlorinated swimming pools may cause exacerbation of asthmatic symptoms even if the level of bilirubin is high. Moreover, our patient had high levels of bilirubin at birth and some studies have found a link between neonatal hyperbilirubinemia and childhood asthma and allergic diseases [7]. In addition, we do not know the effects of HFE mutations on hyperbilirubinemia and asthma. Mutation of HFE genes which are responsible for iron metabolism, leads to increased intestinal iron absorption which results in iron overload and, consequently, hemochromatosis $(\mathrm{HH})$. HFE-related hemochromatosis is the most frequent form of the disease and the most common autosomal recessive disorder in Northern European populations, with a prevalence of $1: 200$ to $1: 250$ for homozygosity and a carrier rate of $1: 8$ to $1: 12$. The symptomatic phenotype preceded by fatigue, arthropathy and impotence, occurs in homozygotic males predominantly between the $4^{\text {th }}$ and $6^{\text {th }}$ decade of life, depending on gene penetration and multiple concomitant factors [8]. The clinical impact of HFE gene carriage is still unknown.

In conclusion, the authors suggest a need for further research to examine whether the hyperbilirubinemia (e.g. Gilbert syndrome and/or hemochromatosis) has a protective or toxic effect on pulmonary function (in particular the effect on airway inflammation in asthma).

\section{Conflict of interest}

The authors declare no conflict of interest.

\section{References}

1. Nakagami T, Toyomura K, Kinoshita T, Morisawa S. A beneficial role of bile pigments as an endogenous tissue protector: anti-complement effects of biliverdin and conjugated bilirubin. Biochim Biophys Acta 1993; 1158: 189-93.

2. Wang HD, Yamaya M, Okinaga S, et al. Bilirubin ameliorates bleomycin-induced pulmonary fibrosis in rats. Am J Respir Crit Care Med 2002; 165: 406-11.

3. McCarty MF. "Iatrogenic Gilbert syndrome" - a strategy for reducing vascular and cancer risk by increasing plasma unconjugated bilirubin. Med Hypotheses 2007; 69: 974-94.

4. Curjuric I, Imboden M, Adam M, et al. Serum bilirubin is associated with lung function in a Swiss general population sample. Eur Respir J 2014; 43: 1278-88.

5. Horsfall LJ, Rait G, Walters K, et al. Serum bilirubin and risk of respiratory disease and death. JAMA 2011; 305: 691-7.

6. Horsfall LJ, Hardy R, Wong A, et al. Genetic variation underlying common hereditary hyperbilirubinaemia (Gilbert's syndrome) and respiratory health in the 1946 British birth cohort. J Hepatol 2014; 61: 1344-51.

7. Huang L, Bao Y, Xu Z, et al. Neonatal bilirubin levels and childhood asthma in the US Collaborative Perinatal Project, 1959-1965. Am J Epidemiol 2013; 178: 1691-7.

8. Pietrangelo A. Hereditary hemochromatosis-a new look at an old disease. N Eng J Med 2004; 350: 2383-97. 